\title{
Setting Long Distance WiFi Records: Proofing Solutions for Rural Connectivity
}

\author{
Ermanno Pietrosemoli \\ Fundación Escuela Latinoamericana de Redes \\ Universidad de los Andes \\ Mérida - Venezuela \\ <ermanno@ula.ve>
}

\begin{abstract}
802.11 WiFi technology is commonly used for creating wireless networks with a range of about one hundred meters. With careful planning and proper antennas, this same equipment can be used to make point-to-multipoint links of tens of kilometers and point-to-point links of hundreds of kilometers. This paper presents some experiments at distances of up to 382 kilometer that were performed in Venezuela from April 206 to July 2007, as well as an affordable instrument setup for long distance antenna alignment.
\end{abstract}

\section{Introduction}

For developing countries, wireless allows a leapfrogging over the traditional telecommunications infrastructure. This has been proved in many countries of Africa and Latin America, where the number of mobile phones has now surpassed the number of land lines in the last decade of the past century or the first of the current one. Although fiber optics offers much greater bandwidth, and satellite systems are great for unidirectional broadcast services, they cannot compete with land-based radio from a cost perspective.

Furthermore, both fiber optic and satellite systems require large investments and considerable expertise which means that they can only be deployed by large organizations with deep pockets that can wait several years before recovering their investments. Terrestrial microwave systems, on the other hand, are less capital intensive and can be deployed by smaller organizations and even local communities.

Since the early 90 's $\mathrm{s}^{1}$ we have been experimenting with the means for providing Internet access through wireless technology, first using packet radio and later with spread spectrum techniques in our home town of Mérida, Venezuela. These efforts led to the deployment of a wireless network that spans most of the state of Mérida, honored by the organizers of SuperComm 1998 in Atlanta, Georgia, with the SuperQuest Award in the Remote Access category.

With the development of the IEEE 802.11 standard, the cost of wireless data transmission for short distances plummeted and many people around the world started using devices based in this technology for long distance communications. By overlaying Voice over IP (VoIP) on these networks, telephony services can also be offered in rural or underserved areas at a fraction of the cost of wired services. Furthermore, these technologies can be installed by a grassroots community with a moderate amount of technical skills

1 Ermanno Pietrosemoli, "Wireless Data Transmission in the Andes: Networking Merida State”, Inet'99, San Francisco, Ca., U.S.A., 1999. 
Accordingly, WiFi, the sobriquet for 802.11 standard based devices, was used by a group of American radio amateurs to demonstrate transmission at a distance of 125 miles in $2004^{2}$. Thanks to a favorable topography, Venezuela already has some long range WLAN links. Since 2001, Fundacite Mérida has operated a $70 \mathrm{~km}$ link between Pico Espejo and Canaguà ${ }^{3}$. To test the extreme possibilities of long distance WLAN, we have successfully used inexpensive WiFi equipment to establish links at $279 \mathrm{~km}$ and $382 \mathrm{~km}$.

\section{Finding the Sweet Spot}

Technically speaking, to test the limits of this technology, it is necessary to find a path with an unobstructed line-of-sight and a clearance of at least $60 \%$ of the first Fresnel zone. As the distance between sites increases, the curvature of the Earth becomes a serious obstacle, requiring higher elevation at both ends. Installation on towers or other tall structures is mandatory, and the longest distance links are only possible from high elevations.

When searching for an area of terrain in Venezuela with high elevation at the ends and low ground in between, we first focused on the Guayana region. Although plenty of high grounds are to be found, in particular the famous "tepuys" (tall mesas with steep walls), there were always obstacles in the middle ground. Our attention then shifted to the Andes, whose steep slopes (rising abruptly from the plains) proved adequate to our requirements. Pico del Aguila has an altitude of 4200 meters and is about a two hours drive from Mérida. It has clear line-of-sight to the town of El Baúl, in Cojedes State. Using the free software Radio Mobile we found that there was no obstruction for the first Fresnel zone (spanning $280 \mathrm{~km}$ ) between Pico del Aguila and El Baúl (Figure 1).

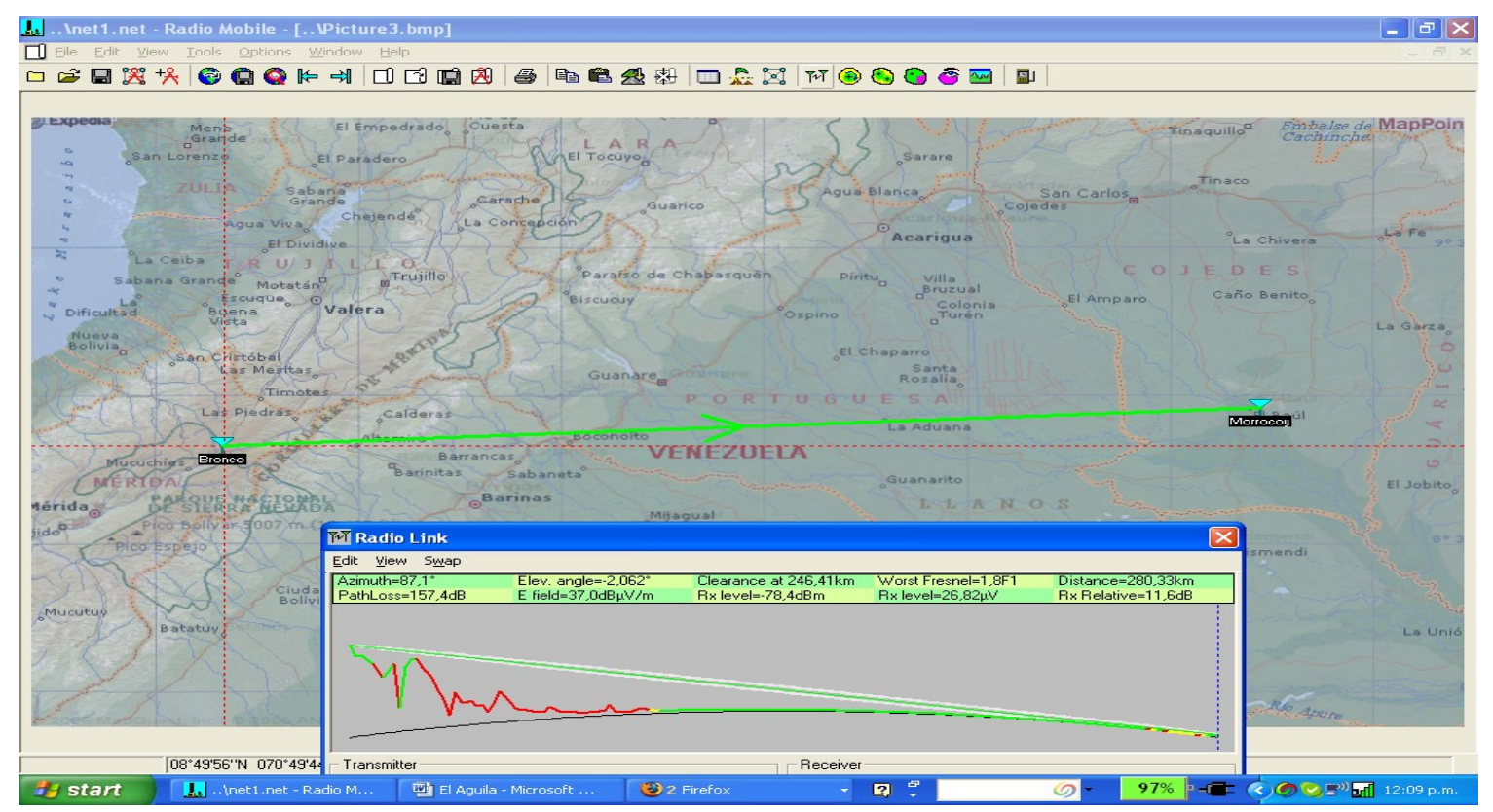

Figure 1: Layout and profile of the $279 \mathrm{~km}$ link between Pico del Aguila and cerro Morrocoy in EI Baul.

\section{Hardware Specifications}

124.9 miles is the official record http://www.wifiworldrecord.com/2005writeup.html Fundacite Merida http:/www.funmrd.gob.ve

Ermanno Pietrosemoili, Case Study: long Distance 802.11, in Flickenger, R. 2008 Wireless Networking in the Developing World 2nd edition, pp355-369 
Once satisfied with the existence of a suitable trajectory, we looked at the equipment needed to achieve our goal of creating a long-distance link. Up to this point, we had been using Orinoco cards for a number of years. Sporting an output power of $15 \mathrm{dBm}$ and a receive threshold of $-84 \mathrm{dBm}$, they are robust and trustworthy. The free space loss at $282 \mathrm{~km}$ is 149 $\mathrm{dB}$, so we would need $30 \mathrm{dBi}$ antennas at both ends and even that would leave very little margin for other losses.

On the other hand, there are the popular Linksys WRT54G wireless routers that can run Linux. The Open Source community has written several firmware versions for it that allow for a complete customization of every transmission parameter. In particular, OpenWRT firmware ${ }^{5}$ allows for the adjustment of the acknowledgment time of the MAC layer, as well as the output power. Another firmware called DD-WRT ${ }^{6}$ has a graphical user interface (GUI) and a very convenient site survey utility. Furthermore, the Linksys can be located closer to the antenna than a laptop; so we decided to use a pair of them. One was configured as an AP (access point) and the other as a client.

The WRT54G can operate at $100 \mathrm{~mW}$ output power with good linearity, and can even be pushed up to $200 \mathrm{~mW}$. But at this value, non linearity is very severe and spurious signals are generated, which should be avoided. Although this is consumer grade equipment and quite inexpensive, after years of using it, we felt confident that it could serve our purpose. Of course, we kept a spare set handy just in case. By setting the output power to $100 \mathrm{~mW}$ (20 $\mathrm{dBm}$ ), we could obtain a $5 \mathrm{~dB}$ power budget advantage compared with the Orinoco card. Therefore, we settled for a pair of WRT54Gs as our preferred option for making this link.

\section{Site Survey}

On January 15, 2006, I went to Pico Águila to check out the site that Radio Mobile had reported as suitable. Technically speaking, the azimuth towards El Baúl is $86^{\circ}$, but since the magnetic declination is $8^{\circ} 16^{\prime}$, the antenna would need to be pointed to a magnetic bearing of $94^{\circ}$.

Unfortunately, line of sight was obstructed by an obstacle in the $94^{\circ}$ direction that had not been shown by the software, due to the limited resolution of the freely available digital elevation maps. After riding my mountain bike for several hours examining the surrounding area, I identified a more suitable location clear of obstacles, south of the main road and a few kilometers from the originally planned site (Figure 2).

\footnotetext{
5 RadioMobile, http://www.cplus.org/rmw/english1.html

6 Abdus Salam ICTP http://wireless.ictp.it
} 


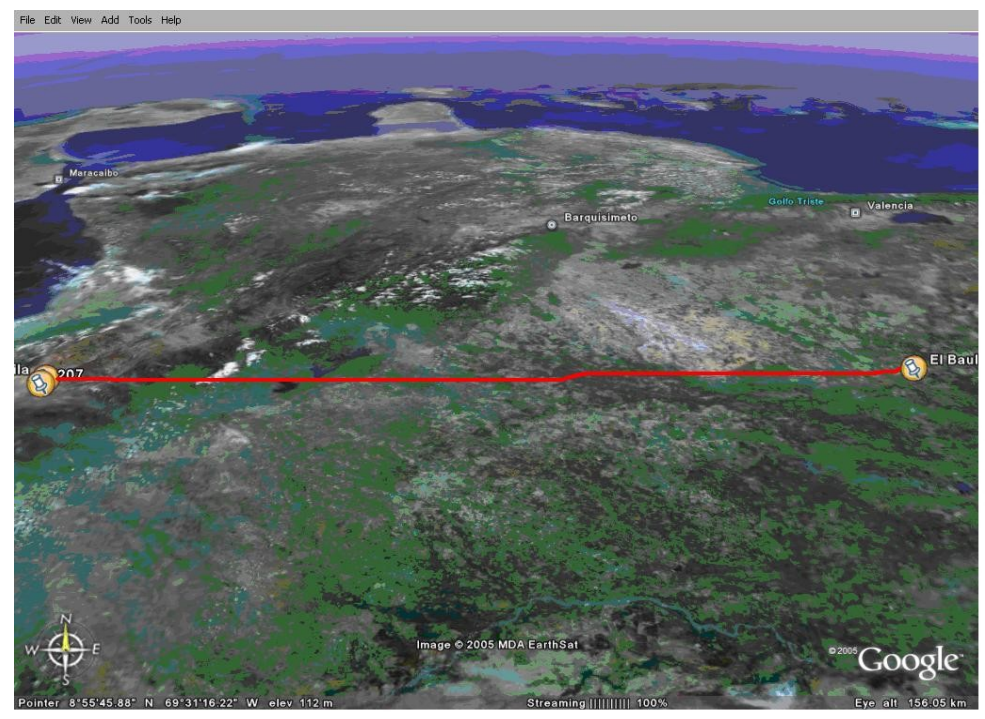

Figure 2: layout of the $279 \mathrm{Km}$ path between Pico del Aguila and El Baul in venezuela

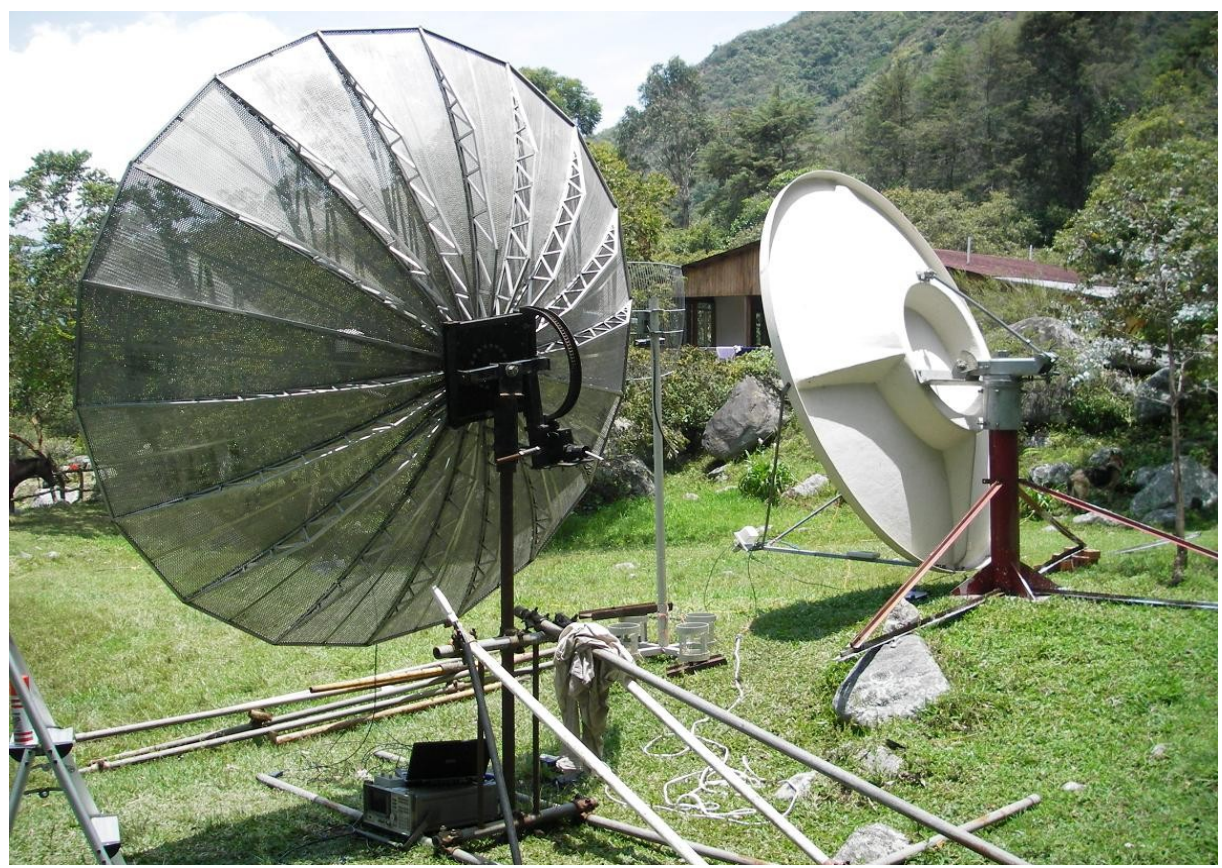

Figure 3: Testing antennas to be deployed at both ends of the link.

\section{Antenna Selection}

High gain antennas for the $2.4 \mathrm{GHz}$ band were not available in Venezuela. As importation costs are considerable, we decided instead to recycle a pair of parabolic reflectors (formerly used for satellite service) and replaced the feed with one designed for $2.4 \mathrm{GHz}$. We proved the concept with an $80 \mathrm{~cm}$ dish. The gain was way too low, so we tried a 2.4 meter reflector with an offset feed. This offered ample gain, albeit with some difficulties in the 
aiming of the $3.5^{\circ}$ beam. The $22.5^{\circ}$ offset meant that the dish appeared to be pointing downwards when it was horizontally aligned as can be seen in Figure 3.

Several tests were performed using various cantennas (homemade antenna built with a can) and a $12 \mathrm{dBi}$ Yagi-Uda as a feed. We pointed the antenna at a base station of the university wireless network that was located $11 \mathrm{~km}$ away on a $3500 \mathrm{~m}$ mountain. The test site sits at $2000 \mathrm{~m}$ and therefore the elevation angle is $8^{\circ}$.

We were able to establish a link with the base station at La Aguada, but our efforts to measure the gain of the setup using Netstumbler, a popular program that reports the strength of the received WiFi signal, were not successful. There was too much fluctuation on the received power values of live traffic. For a meaningful measurement of the gain, we needed a signal generator and spectrum analyzer. These instruments were also required for the field trip in order to align the antennas properly.

On February, 2006 I traveled to Trieste, Italy to partake in the annual wireless training event I have been attending since 1996. While there I mentioned the project to my colleague Carlo Fonda who was immediately thrilled and eager to participate.

The collaboration between the Latin American Networking School (EsLaRed) and the Abdus Salam International Centre for Theoretical Physics (ICTP) in the wireless field goes back to 1992, when the first Networking School was held in Mérida with ICTP support. Since then, several activities in which members of both institutions have participated have taken place, notably the yearly training in wireless networking at ICTP and the ones dedicated to computer networks in general organized by EsLaRed in several countries of Latin America.

Accordingly, it was not difficult to persuade Dr. Sandro Radiciella, the head of the Aeronomy and Radio Propagation Laboratory at ICTP, to support Carlo Fonda's trip in early April to Venezuela in order to participate in the experiment.

Back home I found a $2.75 \mathrm{~m}$ parabolic central fed mesh antenna at a neighbor's house. Mr. Ismael Santos graciously lent his antenna for the experiment. After dismounting and reassembling the parabolic mesh dish, we changed the feed for one that works at $2.4 \mathrm{GHz}$ and aimed the ensemble to a signal generator located on top of a ladder some $30 \mathrm{~m}$ away. With a spectrum analyzer we could measure the maximum of the signal and therefore locate the focus as well as pinpoint the boresight for the antennas, as shown on

We also compared the power of the received signal with the output of a commercial $24 \mathrm{dBi}$ antenna achieving an improvement of $8 \mathrm{~dB}$, which led us to believe that the overall gain of our antenna was about $32 \mathrm{dBi}$. Of course, there is some uncertainty in this value since we were receiving reflected signals as well, but the value agreed with the calculations made from the antenna's dimensions.

Once we were satisfied with the proper functioning and aim of both antennas, we decided to do a site survey at the other end of the El Baúl link. Carlo Fonda, Gaya Fior and I reached the site on April 8th. The following day, we found a hill (south of the town) with two telecom towers from two cell phone operators and one belonging to the mayor of El Baúl.

The hill of Morrocoy is some $75 \mathrm{~m}$ above the surrounding area, about $125 \mathrm{~m}$ above sea level. It provides an unobstructed view towards El Aguila. There is a dirt road to the top, a must for our purpose, given the weight of the antenna. 


\section{Performing the Experiment}

On Wednesday April 12th, 2006 Javier Triviño and I traveled towards Baúl with the offset antenna loaded on top of a four-wheel drive truck. Early on the morning of April 13th, we installed the antenna and pointed it at a compass bearing of $276^{\circ}$ (given that the declination is $8^{\circ}$, the true Azimuth is $268^{\circ}$ ).

At the same time, the other team (composed of Carlo Fonda and Gaya Fior from ICTP, with assistance of Franco Bellarosa, Lourdes Pietrosemoli and José Triviño) rode to the previously surveyed area at Pico del Aguila in a pick-up truck that carried the 2.7 meter mesh antenna (Figure 6):

Poor weather is common at altitudes of $4200 \mathrm{~m}$ above sea level. The team at El Aguila was able to install and point the mesh antenna before the fog and sleet began.

Power for the signal generator was supplied from the truck by means of a $12 \mathrm{VDC}$ to $120 \mathrm{VAC}$ inverter. At $11 \mathrm{am}$ in El Baúl, we were able to observe a $-82 \mathrm{dBm}$ signal at the agreed upon $2450 \mathrm{MHz}$ frequency using the spectrum analyzer. To be sure we had found the proper source, we asked Carlo to switch off the signal. Indeed, the trace on the spectrum analyzer showed only noise. This confirmed that we were really seeing the signal that originated some $280 \mathrm{~km}$ away.

After turning the signal generator on again, we performed a fine tuning in elevation and azimuth at both ends. Once we were satisfied that we had attained the maximum received signal, Carlo removed the signal generator and replaced it with a Linksys WRT54G wireless router configured as an access point. Javier substituted the spectrum analyzer on our end for another WRT54G configured as a client.

At once, we started receiving "beacons" but TCP/IP packets did not get through. This was expected, since the propagation time of the radio wave over a $300 \mathrm{~km}$ link is $1 \mathrm{~ms}$. It takes at least $2 \mathrm{~ms}$ for an acknowledgment to reach the original transmitter. Fortunately, the OpenWRT ${ }^{7}$ firmware allows for adjusting the ACK timing. After Carlo adjusted for the increase in delay above what the standard Wi-Fi link expects, we began receiving ICMP packets with a mean delay time of $5 \mathrm{~ms}$, as can be seen in Figure 4. 


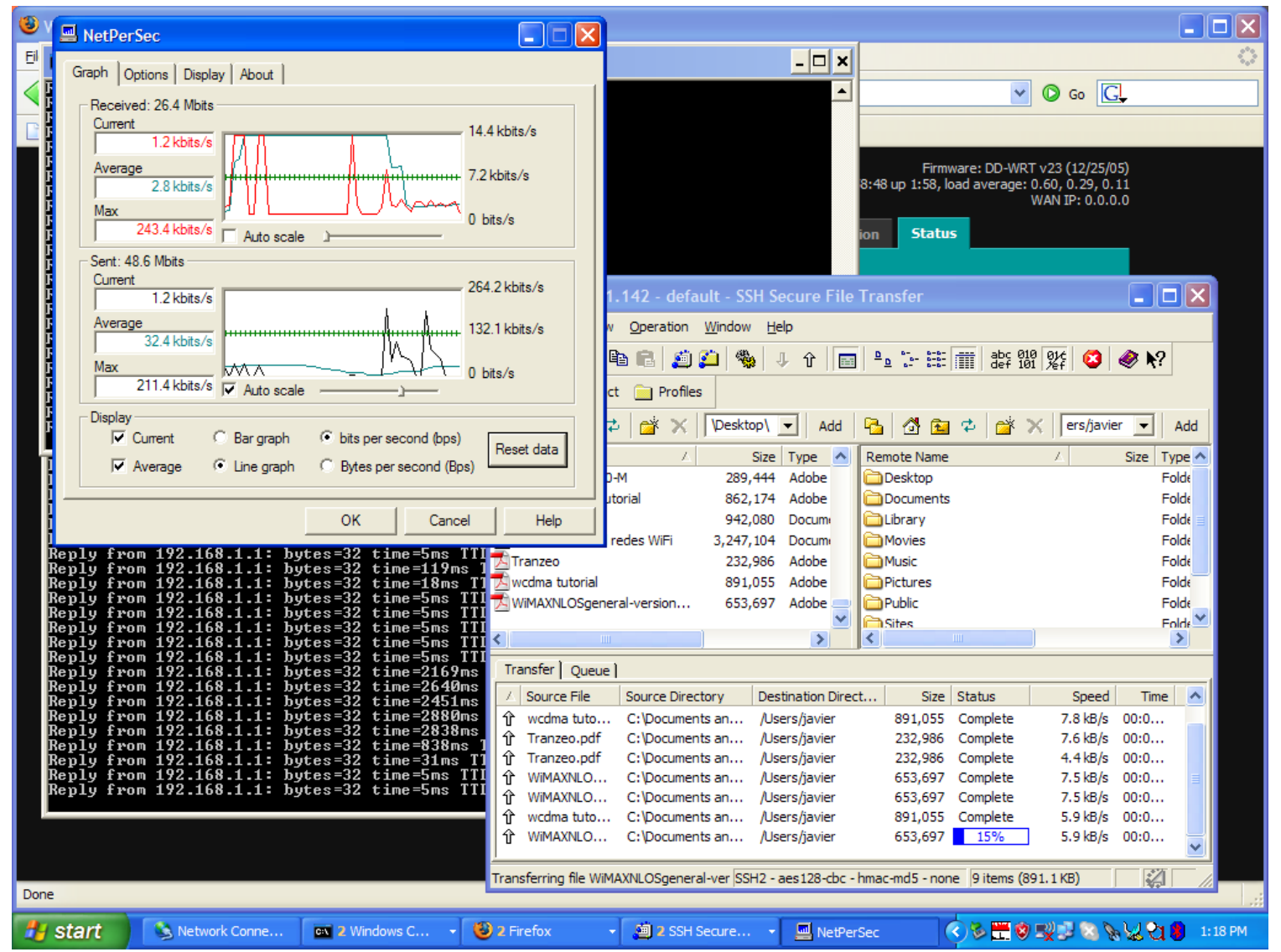

Figure 4: Screenshot of the received signal at EI Baul. $279 \mathrm{~km}$ from El Aguila.

We proceeded to transfer several .PDF files between Carlo's and Javier's laptops, with speeds of about $65 \mathrm{kbps}$.

\section{Improving Performance with TDMA}

One year after performing this experiment, we found the time and resources to repeat itu sing a commercial $30 \mathrm{dBi}$ antenna, and a couple of wireless routers which had been modified by the TIER group led by Dr. Eric Brewer of Berkeley University ${ }^{8}$. The purpose of our modification of the standard WiFi MAC was to make it suitable for long distance applications by replacing the CSMA Media Access Control with TDMA. The latter is better suited for long distance point-to-point links since it does not require the reception of ACKs. This eliminates the need to wait for the $2 \mathrm{~ms}$ round trip propagation time on a $300 \mathrm{~km}$ path.

On April 28th, 2007, a team formed by Javier Triviño, José Torres and Francisco Torres installed one of the antennas at El Aguila site. A solid link was quickly established using the Linksys WRT54G routers. This allowed for video transmission at a measured throughput of $65 \mathrm{kbps}$. With the TDMA routers, the measured throughput was $3 \mathrm{Mbps}$ in each direction. This produced the total of $6 \mathrm{Mbps}$ as predicted by simulations done at Berkeley University by the TIER team. 
Thrilled by these results, which pave the way for really inexpensive long distance broadband links, the second team moved to another location that I had previously identified at $382 \mathrm{~km}$ from El Aguila, in a place called Platillón. Platillón is $1500 \mathrm{~m}$ above sea level and offers an unobstructed first Fresnel zone towards El Aguila (located at $4200 \mathrm{~m}$ above sea level). The Radio Mobile plot is shown in Figure 5.

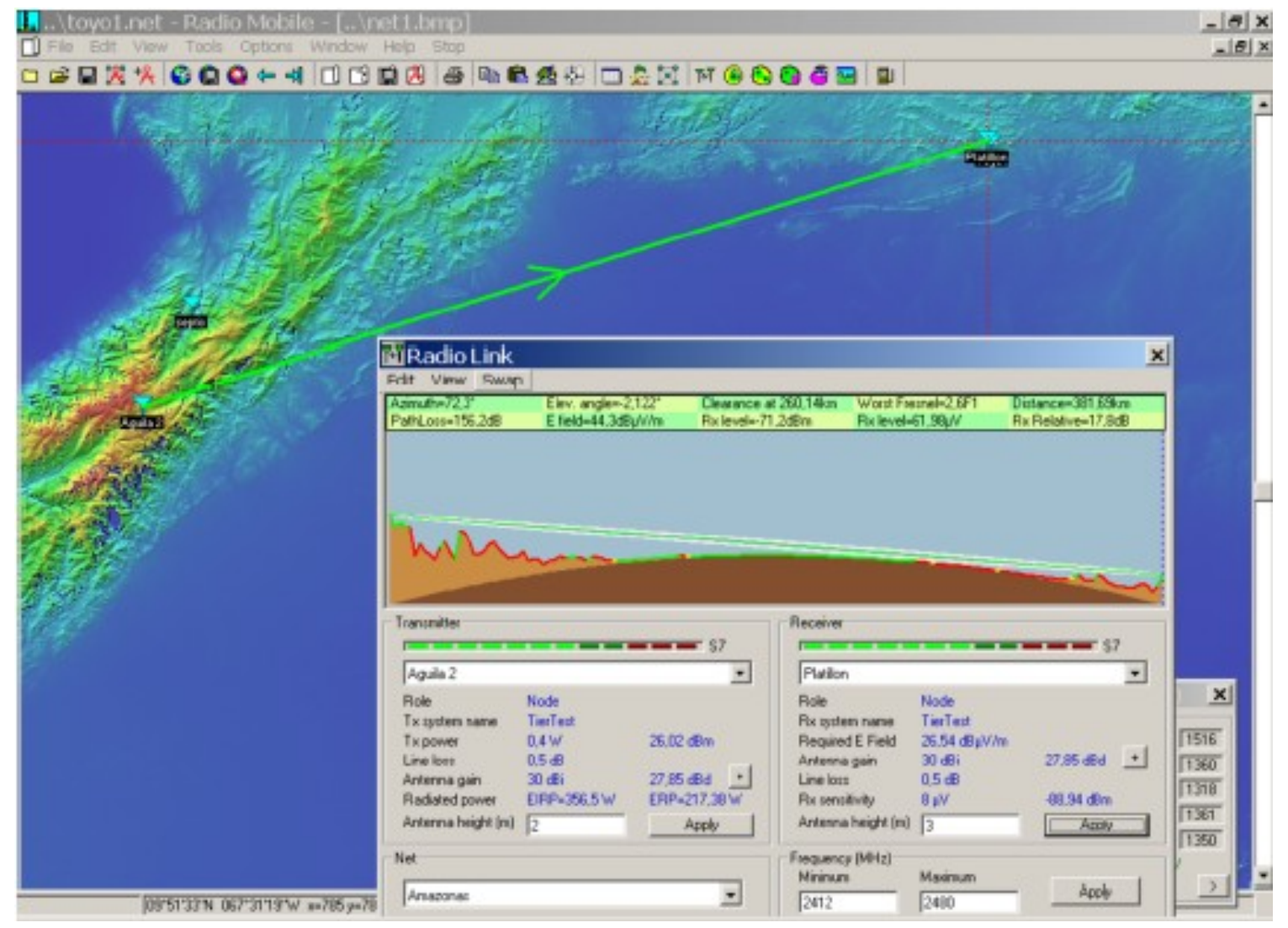

Figure 5: Layout and profile of the $381 \mathrm{~km}$ path.

Again, the link was quickly established with the Linksys and the TIER supplied routers. The Linksys link showed approximately $1 \%$ packet loss, with an average round trip time of $12 \mathrm{~ms}$. The TIER equipment showed no packet loss. This allowed for good quality video transmission, but the link was not stable. We noticed considerable signal fluctuations that often interrupted the communication.

However, when the received signal was sufficient, the measured throughput was a total of 6 Mbps bidirectional with the TIER routers implementing TDMA. So, the preliminary conclusion is that the $280 \mathrm{~km}$ link is stable, and the $380 \mathrm{~km}$ one is probably at the edge of the 2.4 GHz link capabilities with low cost equipment.

\section{Conclusions}

Although further tests must be conducted to ascertain the limits for stable throughput, we are confident that WiFi has a great potential for long distance broadband communication. It is particularly well suited for rural areas where the spectrum is still not crowded and interference is not a problem, provided there is sufficient radio line of sight (clearance of at least $60 \%$ of the first Fresnel zone). 
It is worth noting that the performance obtained on this long distance links shows the viability of WiFi as a low cost alternative to WiMAX for backhaul applications. Similarly, it has also demonstrated the capability of WiFi for point-to-multipoint and even mesh topologies ${ }^{9}$, which makes it the technology of choice for community-based networks for its low cost and limited installation skill requirements, especially in rural areas where the interference problem of unlicensed bands is less severe. In order to make this technology really affordable, an inexpensive method of aligning antennas when the other end of the links is not visible has to be found. The standard method of using a signal generator on one end and a spectrum analyzer on the other is too expensive.

Software based signal strength indicators like netstumbler can be used for short distance links, but they are not adequate for long distance, because the WiFi signal is broadband and it must be relatively strong in order to be decoded by standard WiFi radios. A commercial spectrum analyzer is very sensitive and can effectively measure the received power of a single frequency signal like the one produced by a commercial signal generator, but requires a considerable investment.

WiSpy $^{10}$ is a USB dongle that essentially performs the functions of a spectrum analyzer for the $2.4 \mathrm{GHz}$ band at an affordable price. But we are still missing an inexpensive signal generator. During the Air Jaldi WSFII in Dharamsala ${ }^{11}$, I was pondering this conundrum and Elektra (Corinna Aichele) told me that in Germany they were selling "video senders" - devices meant to transmit analog video signals - which would make for an inexpensive signal generator. After experimenting with many of these cheap concoctions that were transmitting out of band or erratically, I finally found one that can really substitute for a signal generator for antenna alignment purposes because it: a) has an antenna connector that allows for the attachment to the antenna to be deployed; b) allows the selection of 8 different carrier frequencies in the $2.4 \mathrm{GHz}$ band; c) sports a 1 Watt $(30 \mathrm{dBm})$ output power, enough to be detected $300 \mathrm{~km}$ away; d) is small enough and can be powered by any 12 volt source; and, e) is relatively stable in output power and frequency during the time needed to align an antenna. With this, a kit comprising an inexpensive signal generator and a signal strength detector (Figure 6) is finally available for the wireless networking practitioner that allows for the alignment of antennas at long distances.

\footnotetext{
9 http://freifunk.net/wiki/FreifunkFirmwareEnglish

10 http://www.metageek.com

11 http://drupal.airjaldi.com/
} 


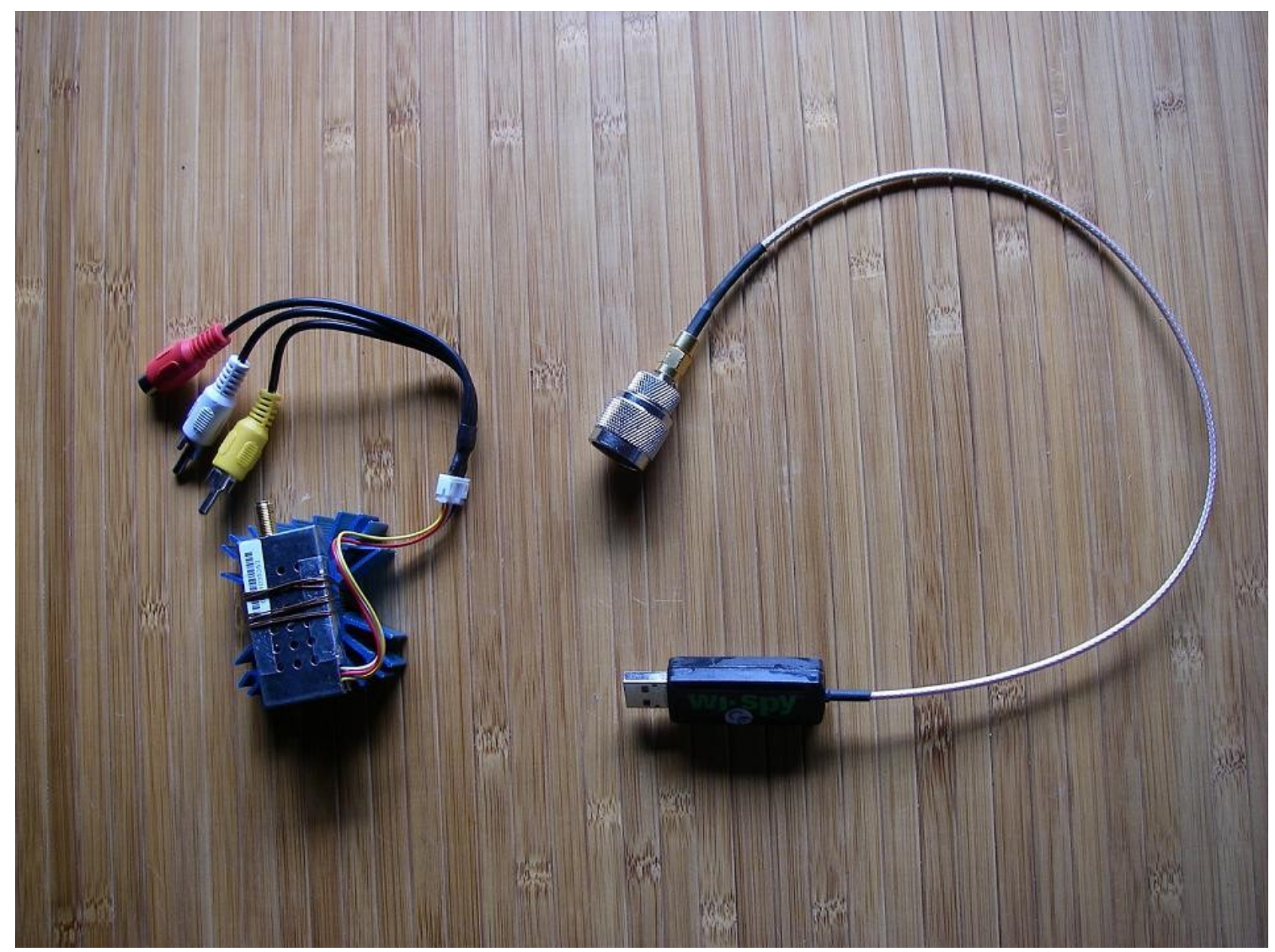

Figure 6: Low cost antenna alignment set: Signal Generator (video sender) and Spectrum Analyzer (WiSpy).

I gratefully acknowledge the funding of this work by ICA-IDRC through the TRICALCAR project. 